\title{
The Application of Non-orthogonal Multiple Access in Wireless Powered Communication Networks
}

\author{
Yi Yuan, Zhiguo Ding \\ School of Computing and Communications Lancaster University, UK \\ Email: \{y.yuan3, z.ding\}@lancaster.ac.uk
}

\begin{abstract}
This work investigates the application of nonorthogonal multiple access (NOMA) scheme for the uplink of wireless powered communication networks (WPCN). We maximize the sum rate by jointly designing of the time allocation, the downlink energy beamforming and receiver beamforming. To solve this nonconvex problem, a method of two stages optimization is proposed. In the first stage, we apply the series approximations to obtain the optimal energy beamforming and receive beamforming with fixed time allocation. In the second stage, one-dimensional search is considered to obtain the optimal time allocation. For the approximation part, successive convex approximation (SCA) method is used to convert a mixed integer non-linear program into its convex approximation problem. The numerical results illustrate that the proposed algorithm converges to the local optimum solution and the proposed scheme outperforms the fixed time allocation scheme and orthogonal multiple access (OMA).
\end{abstract}

Index Terms-Wireless power transfer, non-orthogonal multiple access, successive convex approximation.

\section{INTRODUCTION}

Recently, non-orthogonal multiple access (NOMA) has been considered as a promising enable technique for the fifth generation $(5 \mathrm{G})$ networks due to its superior spectral efficiency [1]. The fundamental difference between NOMA and orthogonal multiple access (OMA) schemes, e.g., time/frequency/code division multiple access (TDMA/FDMA/CDMA) is that NOMA applies power domain to achieve multiple access simultaneously. For this reason, successive interference cancellation (SIC) strategy could be applied at the receiver to improve the spectral efficiency [2]-[6], [11]. The performance of downlink NOMA with randomly deployed users was investigated in [2], the application of multiple-input multiple-output (MIMO) NOMA with fixed and more sophisticated power allocation schemes also was derived in [3]. Ding et al., analyzed the outage probability and diversity gain in proposed cooperative NOMA scheme [4]. Meanwhile, an uplink NOMA scheme with single and multiple transmission antennas was proposed in [5] and [6].

In addition to NOMA, which improves the spectral efficiency through more efficient spectrum usage, energy harvesting has been considered as a promising solution to prolong the lifetime of wireless devices. Different from the traditional harvesting way, wireless power transfer from radio frequency (RF) signal has rekindled the interest of researchers [7]-[11]. L. Varshney introduces that both information and energy could be extracted from the same RF signal at the same time in wireless powered communication network (WPCN) [7]. Based on this theory, two practical receiver architectures were proposed in a MIMO system [8]. A typical usage of WPCN model is that nodes use harvested power to charge their batteries and transmit their information to a base station (BS). However, receivers cannot harvest energy and transmit information simultaneously in practical. A harvest - then - transmit protocol was proposed in [9], [10] and [11] to overcome this problem. In [9], time division multiple access (TDMA) is considered for the uplink information transmission, while space-divisionmultiple-access (SDMA) is used based on multi-antenna at the BS [10]. With the similar set up to [9], authors apply this protocol to implement that nodes first harvest energy and then transmit their information in the uplink NOMA scheme [11]. Through the comparison with TDMA proposed in [9], NOMA in [11] might improve fairness and minimum date rate.

In this work, we extend the investigate of [11] to a multiantenna WPCN system, which consists of one multi antenna BS and several powerless users. The goal is to maximize sum rate by jointly designing of the time allocation, energy beamforming and receiver beamforming. The optimization problem is NP-hard problem, which is difficult to be solved. In order to solve this problem, we consider a method of two stages. In the first stage, we proposed an algorithm to find the optimum energy beamforming and receiver beamforming, based on the method of successive convex approximation (SCA) in [12]. The key idea of this algorithm is converting a mixed integer non-linear program into its convex approximation. In the second stage, we consider one-dimensional search to obtain the optimum time allocation with the optimal solution in step one. The numerical results illustrate that the proposed algorithm converges to the optimal local point within a few iterations.

\section{System ModeL}

Consider a WPCN consisting of one multi-antenna BS $M>1$ and $\mathrm{K}$ single-antenna users. We assume that the same Rayleigh fading channels are used for the downlink energy transfer and uplink information transfer. Hence, the channel from the BS to the $k$-th user can be denoted by the conjugated complex vectors $\boldsymbol{h}_{k} \in \mathbb{C}^{M \times 1}$, zero mean and unit variance, during each block transmission time $T$. It assumes that the distance between each user and BS increases as the index of each user grows. We also assume that the far user suffer more pass loss and has the worse channel condition.

Due to the assumption of no available power at all users, they need replenish the energy from BS to transmit their 
independent information based on the "harvest-then-transmit" protocol proposed in [9]. Each block is divided into two parts. In the first part, $\tau T(0<\tau<1)$, BS broadcast wireless energy to all users, and the remain part, $(1-\tau) T$, is assigned to users to transmit information simultaneously by employing NOMA scheme. For convenience, we assume time duration $T=1$ in this paper.

During the downlink phase, BS broadcast energy to all users. Since a far user from the BS receives less power in the downlink and suffers more pass loss in the uplink than a near user, the BS applies different energy beamforming weights to control the power allocation. Then, the harvested energy by $j$-th user is given by

$$
E_{j}=\sum_{i=1}^{K} \xi_{j} \tau T\left|\boldsymbol{h}_{j}^{H} \boldsymbol{w}_{i} s_{i}^{d l}\right|^{2}, \quad j=1, \cdots, K,
$$

where $s_{i}^{d l}$ is an independent and identically distributed (i.i.d) random variable with zero mean and unit variance, $E\left[\left|s^{d l}\right|^{2}\right]=$ $1, \boldsymbol{w}_{i}$ and $0<\xi_{j}<1$ denote energy beamforming and energy harvesting efficiency, respectively. For convenience, we set $\xi_{1}=\cdots=\xi_{K}=1$ in this paper. Then the available average transmit power of $j$-th user to transmit its information in the uplink phase can be expressed as

$$
Q_{j}=\frac{\sum_{i=1}^{K} \tau\left|\boldsymbol{h}_{j}^{H} \boldsymbol{w}_{i}\right|^{2}}{1-\tau}, \quad j=1, \cdots, K .
$$

In the uplink phase, the transmit signal of $j$-th user can be expressed as

$$
x_{j}=\sqrt{p_{j}} s_{j}^{u l}, \quad j=1, \cdots, K,
$$

where $s_{j}^{u l}$ denotes the information-carrying signal, which is also assumed to be i.i.d random variables with zero mean and unit variance, and $p_{j}$ denotes the used power, which is bounded by the available transmit power $Q_{j}$. We assume to use linear receiver at BS to decode the user's information. The receiver beamforming vector $\boldsymbol{v}_{k} \in \mathbb{C}^{M \times 1}$ can be used to decode the information of the $k$ th user. The observations for the $k$ th user at the BS can be written as follows

$$
\boldsymbol{v}_{k}^{H} \boldsymbol{y}_{k}=\sum_{j=1}^{K} \boldsymbol{v}_{k}^{H} \boldsymbol{g}_{j} x_{j}+\boldsymbol{v}_{k}^{H} \boldsymbol{n} .
$$

In the following part, we need to define the sum-throughput of this system. According to [5] and [6], any arbitrary decoding order cannot affect the sum-throughput in multiple access system. Hence, two simple fixed decoding order, ascending order and descending order, can be assumed in this paper based on the users' indices. According to assumption of the channel condition of each user in the front of this section, we adopt descending decoding order to increase fairness. In this decoding order scheme, the first decoded user need to endure the whole inter-user interference from other users; the user who has the later decoding order can benefit the throughput by cancelling the interference; the weakest user's message is decoded without any interference. Using Shannons capacity formula, the throughput of the $k$ th user, $1 \leq k \leq(K-1)$, is given by [6]

$$
R_{k}=(1-\tau) \log \left(1+\frac{p_{k}\left|\boldsymbol{v}_{k}^{H} \boldsymbol{h}_{k}\right|^{2}}{\sum_{j=k+1}^{K} p_{j}\left|\boldsymbol{v}_{k}^{H} \boldsymbol{h}_{j}\right|^{2}+\sigma^{2}\left\|\boldsymbol{v}_{k}\right\|^{2}}\right)
$$

while the throughput of $K$ th user can be expressed as

$$
R_{K}=(1-\tau) \log \left(1+\frac{p_{K}\left|\boldsymbol{v}_{K}^{H} \boldsymbol{h}_{K}\right|^{2}}{\sigma^{2}\left\|\boldsymbol{v}_{K}\right\|^{2}}\right) .
$$

Therefore, sum-throughput achieved by NOMA is written as

$$
R_{\text {sum }}=\sum_{k=1}^{K} R_{k}
$$

Note that the whole throughput of system equal to zero if time allocation $\tau$ equal to zero or one.

\section{PRoblem Formulation}

In this section, we aim to maximize sum rate of this system by jointly downlink energy beamforming and uplink receive beamforming as well as time allocation. Thus, the optimization problem is proposed subject to the individual user rate constraint as well as power constraint as follows

$$
\begin{array}{ll}
\max _{\boldsymbol{w}, \boldsymbol{v}, \boldsymbol{p}, \tau} & R_{\text {sum }} \\
\text { s.t. } & \log \left(1+\frac{p_{k}\left|\boldsymbol{v}_{k}^{H} \boldsymbol{h}_{k}\right|^{2}}{\sum_{j=k+1}^{K} p_{j}\left|\boldsymbol{v}_{k}^{H} \boldsymbol{h}_{j}\right|^{2}+\sigma^{2}\left\|\boldsymbol{v}_{k}\right\|^{2}}\right) \geq \frac{R_{\text {min }}}{1-\tau}, \forall_{k}, \\
& p_{k} \leq \frac{\sum_{i=1}^{K} \tau\left|\boldsymbol{h}_{k}^{H} \boldsymbol{w}_{i}\right|^{2}}{1-\tau}, \quad \forall_{k}, \\
& \sum_{i=1}^{K}\left\|\boldsymbol{w}_{i}\right\|^{2} \leq P_{\text {sum }}, \\
& 0<\tau<1
\end{array}
$$

Problem (8) is non-convex due to coupled variables in the objective function and constraints. We proposed a two step method to solve this problem. In the first stage, we focus on following optimization problem to find optimum energy beamforming and receiver vector with given $\tau$

$$
\begin{aligned}
& \max _{\boldsymbol{w}, \boldsymbol{v}, \boldsymbol{p}} R_{\text {sum }} \\
& \text { s.t. }(8 b),(8 c),(8 d) .
\end{aligned}
$$

In the second stage, we consider one-dimensional search to find optimum time allocation over $0<\tau<1$ with the optimal $\boldsymbol{w}^{*}$ and $\boldsymbol{v}^{*}$ as follows

$$
R^{*}=\max _{0<\tau<1}(1-\tau) \log \left(1+g\left(\boldsymbol{w}^{*}, \boldsymbol{v}^{*}, \tau\right)\right) .
$$

It shows that problem (9) is still non-convex and is a NPhard problem, which is difficult to get the globally optimal solution, since it is an instance of mixed-integer non-linear program [13]. Therefore, we proposed an approximation algorithm to solve this problem locally based on the framework of 
SCA [12]. First, we introduce a vector $t \in \mathbb{R}_{+}^{N}$ to relax the objective function in (9) as follows

$$
\begin{aligned}
\max _{\boldsymbol{w}, \boldsymbol{v}, \boldsymbol{p}, \boldsymbol{t}} & \sum_{k=1}^{K}(1-\tau) \log t_{k} \\
\text { s.t. } & t_{k} \leq 1+\frac{p_{k}\left|\boldsymbol{v}_{k}^{H} \boldsymbol{h}_{k}\right|^{2}}{\sum_{j=k+1}^{K} p_{j}\left|\boldsymbol{v}_{k}^{H} \boldsymbol{h}_{j}\right|^{2}+\sigma^{2}\left\|\boldsymbol{v}_{k}\right\|^{2}}, \forall_{k}, \\
& (8 b),(8 c),(8 d) .
\end{aligned}
$$

The equivalence between (9) and (11) can be prove when (11b) holds equality at the optimum for all $k$. Based on the convexity and monotonicity of the logarithm function, (11a) can be expressed as the geometric mean function.

In the next step, we pay more attention to solve the nonconvex constraints $(11 \mathrm{~b}),(8 \mathrm{~b})$ and $(8 \mathrm{c})$. The right side of (11b) is a fractional function with coupled variables. Normally, alternative algorithm can be used to solve this problem by fixed one to optimal others. However, this algorithm is more complex. Therefore, we consider to use difference of convex (DC) function to represent non-convex constraint as two difference of convex functions, because every continuous function can be presented as DC with suitable approximations. By introducing a new variable, (11b) can be equivalent to two inequalities as follows

$$
\begin{aligned}
& \left(t_{k}-1\right) \beta_{k} \leq p_{k}\left|\boldsymbol{v}_{k}^{H} \boldsymbol{h}_{k}\right|^{2}, \\
& \sum_{j=k+1}^{K} p_{j}\left|\boldsymbol{v}_{k}^{H} \boldsymbol{h}_{j}\right|^{2}+\sigma^{2}\left\|\boldsymbol{v}_{k}\right\|^{2} \leq \beta_{k},
\end{aligned}
$$

It is obviously show that above two constraints are still nonconvex. By using the same way, (12a) can be expressed as

$$
\begin{array}{ll}
\left(t_{k}-1\right) \beta_{k} \leq p_{k} a_{k}, & \forall_{k}, \\
\left|\boldsymbol{v}_{k}^{H} \boldsymbol{h}_{k}\right|^{2} \geq a_{k}, & \forall_{k},
\end{array}
$$

and $(12 b)$ is rewritten to two inequalities

$$
\begin{aligned}
& \sum_{j=k+1}^{K} p_{j} b_{j}+\sigma^{2}\left\|\boldsymbol{v}_{k}\right\|^{2} \leq \beta_{k}, \quad \forall_{k}, \\
& \left|\boldsymbol{v}_{k}^{H} \boldsymbol{h}_{j}\right|^{2} \leq b_{j}, \quad j=k+1, \forall_{k} .
\end{aligned}
$$

We note that from (13a) to (14a) are non-convex and (14b) can be formulated as a second order cone (SOC) constraint [14]. In order to approximate (13a) and (14a), we use mathematic approach to reformulate both inequalities as follows

$$
\begin{aligned}
& \left(t_{k}-1+\beta_{k}\right)^{2}+\left(p_{k}-a_{k}\right)^{2} \leq\left(t_{k}-1-\beta_{k}\right)^{2}+\left(p_{k}+a_{k}\right)^{2}, \forall_{k}(15 \\
& \sum_{j=k+1}^{K}\left(p_{j}+b_{j}\right)^{2}-4\left(\beta_{k}-\sigma^{2}\left\|\boldsymbol{v}_{k}\right\|^{2}\right) \leq \sum_{j=k+1}^{K}\left(p_{j}-b_{j}\right)^{2}, \forall_{k} \cdot(15
\end{aligned}
$$

It obviously shows that both side of (15a) and (15b) are convex, but (15a) and (15b) are not jointly convex. Based on the convexity of both side of (15a) and (15b), we introduce Taylor series expansion to approximate the right side of (15a) and (15b) [12]. By applying the first order approximation, the right side of (15a) can be approximated as

$$
\begin{aligned}
& \left(t_{k}-1-\beta_{k}\right)^{2}+\left(p_{k}+a_{k}\right)^{2} \geq \\
& \left(t_{k}^{(n)}-1-\beta_{k}^{(n)}\right)^{2}+2\left(t_{k}^{(n)}-1-\beta_{k}^{(n)}\right)\left(t_{k}-t_{k}^{(n)}-\beta_{k}+\beta_{k}^{(n)}\right)+ \\
& \left(p_{k}^{(n)}+a_{k}^{(n)}\right)^{2}+2\left(p_{k}^{(n)}+a_{k}^{(n)}\right)\left(p_{k}-p_{k}^{(n)}+a_{k}-a_{k}^{(n)}\right) \triangleq \\
& f^{(n)}\left(t_{k}, \beta_{k}, p_{k}, a_{k}\right),
\end{aligned}
$$

and the right side of (15b) is given by

$$
\begin{aligned}
& \sum_{j=k+1}^{K}\left(p_{j}-b_{j}\right)^{2} \geq \\
& \sum_{j=k+1}\left(\left(p_{j}^{(n)}-b_{j}^{(n)}\right)^{2}+2\left(p_{j}^{(n)}-b_{j}^{(n)}\right)\left(p_{j}-p_{j}^{(n)}-b_{j}+b_{j}^{(n)}\right)\right) \triangleq \\
& f^{(n)}\left(p_{j}, b_{j}\right),
\end{aligned}
$$

where $n$ denotes the $n$th iteration. The right side of (16) and (17) are the first order approximation around the point $\left(t_{k}^{(n)}, \beta_{k}^{(n)}, p_{k}^{(n)}, a_{k}^{(n)}\right)$ and $\left(p_{j}^{(n)}, b_{j}^{(n)}\right)$. We can iteratively replace the right side of (15a) and (15b) by the right side of (16) and (17) to obtain a convex approximation. Next, we consider to modify (13b) as follows

$$
\begin{aligned}
& x_{k}^{2}+y_{k}^{2} \geq a_{k}, \quad \forall_{k}, \\
& x_{k}=\Re\left(\boldsymbol{v}_{k}^{H} \boldsymbol{h}_{k}\right), \quad y_{k}=\Im\left(\boldsymbol{v}_{k}^{H} \boldsymbol{h}_{k}\right), \quad \forall_{k} .
\end{aligned}
$$

Note that the set of constraints in (18b) are convex and linear, but (18a) are not. We use SCA to approximate (18a) by introducing $\boldsymbol{u}_{k}=\left(x_{k}, y_{k}\right)^{T}$, then we approximate the left side of (18a) as

$x_{k}^{2}+y_{k}^{2}=\left\|\boldsymbol{u}_{k}\right\|^{2} \geq\left\|\boldsymbol{u}_{k}^{(n)}\right\|^{2}+2\left(\boldsymbol{u}_{k}^{(n)}\right)^{T}\left(\boldsymbol{u}_{k}-\boldsymbol{u}_{k}^{(n)}\right) \geq a_{k}$,

where $\boldsymbol{u}_{k}^{(n)}$ is the linearization point. Through serial modification, the constraint (11b) becomes convex. Due to the similar formate of $(8 b)$ and $(11 b)$, we can use the same approximation method to modify $(8 b)$ as follows

$$
\begin{aligned}
& \left(p_{k}-a_{k}\right)^{2}+4\left(e^{\left(\frac{R_{\min }}{1-\tau}\right)}-1\right) \lambda_{k} \leq \\
& \quad\left(p_{k}^{(n)}+a_{k}^{(n)}\right)^{2}+2\left(p_{k}^{(n)}+a_{k}^{(n)}\right)\left(p_{k}-p_{k}^{(n)}+a_{k}-a_{k}^{(n)}\right),
\end{aligned}
$$

$$
\begin{aligned}
& \sum_{j=k+1}^{K}\left(p_{j}+b_{j}\right)^{2}-4\left(\lambda_{k}-\sigma^{2}\left\|\boldsymbol{v}_{k}\right\|^{2}\right) \leq \\
& \quad \sum_{j=k+1}\left(\left(p_{j}^{(n)}-b_{j}^{(n)}\right)^{2}+2\left(p_{j}^{(n)}-b_{j}^{(n)}\right)\left(p_{j}-p_{j}^{(n)}-b_{j}+b_{j}^{(n)}\right)\right)
\end{aligned}
$$

Finally, we consider to solve non-convex transmission power constraint $(8 \mathrm{c})$. With the similar approximation method of solving (11b), (8c) can be reformulated as follows

$$
p_{k} \leq \sum_{i=1}^{K} \frac{\tau}{1-\tau}\left(\left\|\boldsymbol{\theta}_{k}^{(n)}\right\|^{2}+2\left(\boldsymbol{\theta}_{k}^{(n)}\right)^{T}\left(\boldsymbol{\theta}_{k}-\boldsymbol{\theta}_{k}^{(n)}\right)\right), \forall_{k},
$$




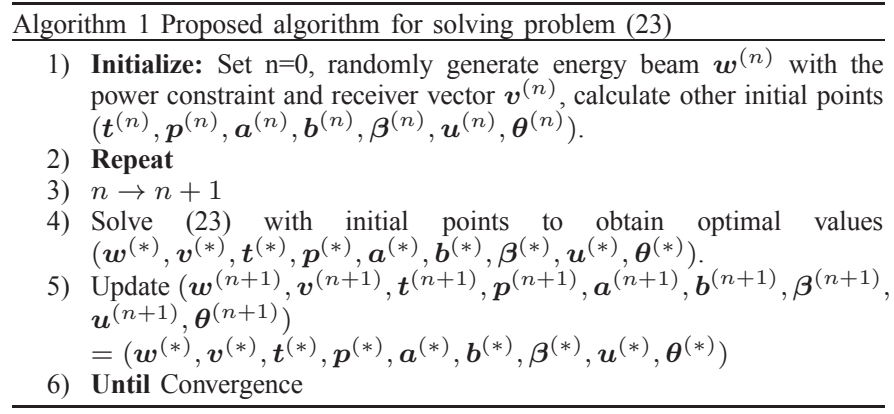

where $\boldsymbol{\theta}_{k}=\left(\Re\left(\boldsymbol{h}_{k}^{H} \boldsymbol{w}_{i}\right), \Im\left(\boldsymbol{h}_{k}^{H} \boldsymbol{w}_{i}\right)\right)^{T}$. Based on the above equivalent transformations and approximations, we can approximate (9) at iteration $n$ by the following convex problem

$$
\begin{aligned}
& \max _{\boldsymbol{w}, \boldsymbol{v}, \boldsymbol{t}, \boldsymbol{p}, \boldsymbol{\beta}, \boldsymbol{a}, \boldsymbol{b}, \boldsymbol{u}, \boldsymbol{\theta}, \boldsymbol{\lambda}} \sum_{k=1}^{K}(1-\tau) \log t_{k} \\
& \text { s.t. }\left(t_{k}-1+\beta_{k}\right)^{2}+\left(p_{k}-a_{k}\right)^{2} \leq f^{(n)}\left(t_{k}, \beta_{k}, p_{k}, a_{k}\right) \\
& \sum_{j=k+1}^{K}\left(p_{j}+b_{j}\right)^{2}-4\left(\beta_{k}-\sigma^{2}\left\|\boldsymbol{v}_{k}\right\|^{2}\right) \leq f^{(n)}\left(p_{j}, b_{j}\right) \\
&(8 d),(14 b),(19),(20),(21),(22) .
\end{aligned}
$$

We update variables for the next iteration by solving (23) until convergence. We outline the proposed iterative algorithm in Algorithm 1.

Convergence Anaylsis: Let $\psi^{(n)}$ and $A^{(n)}$ denote the return optimal objective value and the set of all optimal variables at iteration $n$ of Algorithm 1, respectively. According to the result in [15], the optimal solutions in set $A^{(n)}$ at iteration $n$ are also feasible for optimization problem (23) at iteration $n+1$ due to the linear approximation applying into (16)(22). Therefore, the objective value obtained at iteration $n+1$ is larger than or equal to iteration $n$, because Algorithm 1 generate a nondecreasing sequence of objective values. Meanwhile, the objective value of (23) is also bounded by the power constraint (8d). Thus, Algorithm 1 is guaranteed to converge to some local optimum solution. Based on the Theorem and propositions presented in [12] and [15], it is possible to prove that convergent points of (23) is also satisfied Karush-Kuhn-Tucker (KKT) conditions of the problem (11).

\section{Numerical Results}

In this section we investigate numerical performance evaluation of the proposed algorithm. We consider BS is equipped with $M=4$ antennas, and there are $K=4$ users with single antenna. All users randomly drop on the interval 1 $50 \mathrm{~m}$ from the BS. We assume that the channe of $k$ th user is $\boldsymbol{h}_{k}=\sqrt{d_{k}^{-\gamma}} \hat{\boldsymbol{h}}_{k}$, where $\hat{\boldsymbol{h}}_{k} \sim \mathcal{C N}(0, \boldsymbol{I})$. The pass loss factor sets $\gamma=2$. Furthermore, the energy harvesting efficiency of each user is assumed to be one, $\xi=1$, and the variance of noise is taken to be unity. The target rate $R$ is obtained through solving maximize minimum individual rate problem. The CVX package is used for the simulations [16].
In Fig. 1 we investigate the impact of time allocation on the sum rate with three different transmission power cases. From the figure, it shows that there is a extreme point for each curve. The reason is that the function of sum rate in (8) is a concave function. It is also observed that sum rate first increases with $\tau$ when $\tau<\tau^{*}$, but decreases with increasing $\tau$ when $\tau>\tau^{*}$, where $\tau^{*}$ is the optimal time allocation to maximize the sum rate. This can be explained as follows. When $\tau$ is small, the amount of energy harvested by each user is small. Thus, the more downlink energy transfer time the more available transmit power can be used by each user. However, as $\tau$ becomes larger than $\tau^{*}$, the sum rate reduces more significantly. Because the allocated uplink transmission time becomes smaller with increasing $\tau$. Meanwhile, system needs less energy transfer time with increasing the transmission power.

In the second experiment, we illustrate the convergence performance of the proposed algorithm with different initial points and antennas. The returned successive values of the sum rate is applied as a stopping criteria. When the difference between two consecutive values of the sum rate is less than or equal to $10^{-3}$, the proposed algorithm come out from the iteration loop. As can be seen in Fig.2, the approximated sum rate increases monotonically in the first several iterations, but it converges to the same value within a few of iterations in all cases with this criterion. The system with 4 transmit antenna obtains better sum rate than 2 transmit antenna. This is a result of more transmit antenna can generate more diversity.

In Fig. 3, we present the relationship between the sum rate and total transmit power. We compare the performance of four design methods as follow, the proposed NOMA with/without optimal time allocation, OMA method and SDMA method presented in [10]. It can be seen that, the sum rate increases monotonically with the growth of transmit power for all methods. The proposed NOMA with optimal time allocation has similar performance with SDMA method and gets a better performance gain than another two method. In particular, the performance gap between the proposed algorithm and OMA method becomes larger when the transmit power increases, because OMA method need more transmission time slots to finish information transfer. Besides, we also learn that NOMA employed at the BS can significantly improve the spectrum usage.

\section{CONCLUSION}

In this paper, the sum rate maximization problem is investigated in the uplink NOMA system based on WPCN. Since the problem is nonconvex and NP-hard, we propose a method of two stages to solve this problem. An iterative algorithm is proposed to deal with the double variables by using a serial approximation method in the first stage. Onedimensional search is presented in second stage to obtain the optimal time allocation. The numerical results illustrate that the proposed algorithm converges to the local optimum solution and the proposed scheme outperforms the fixed time allocation scheme and OMA scheme. 


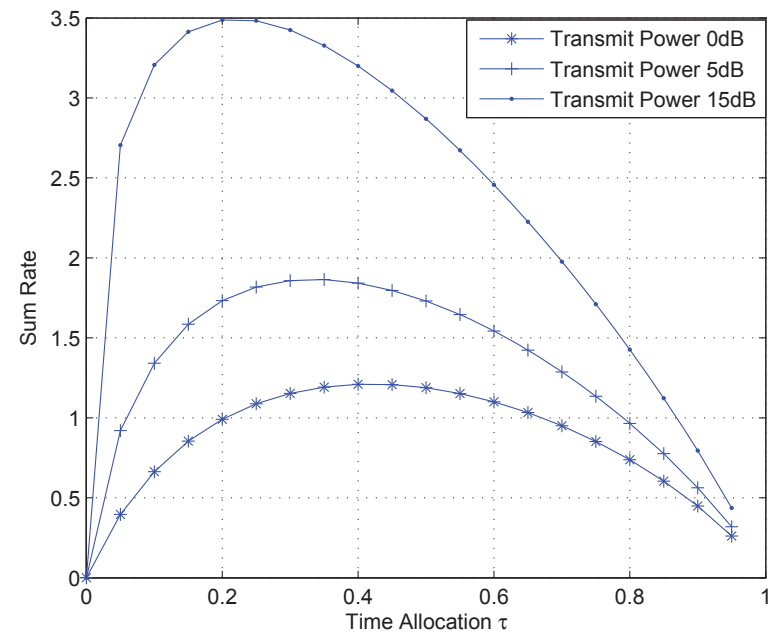

Fig. 1. Sum Throughput versus the time allocation.

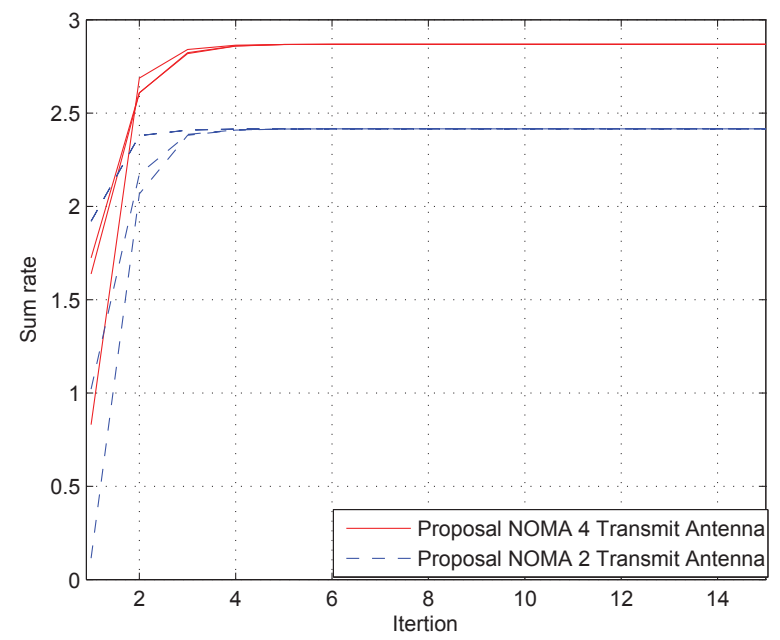

Fig. 2. Convergence behavior of Algorithm 1 with different initial points over different transmit antenna. $\mathrm{K}=4$.

\section{REFERENCES}

[1] Y. Saito, A. Benjebbour, Y. Kishiyama, and T. Nakamura, "Systemlevel performance evaluation of downlink non-orthogonal multiple access (NOMA)," in Proc. IEEE Intl. Symp. Personal, Indoor and Mobile Radio Communications (PIMRC), London, U.K., pp. 611615, Sep. 2013.

[2] Z. Ding, Z. Yang, P. Fan, and H. V. Poor, "On the performance of nonorthogonal multiple access in $5 \mathrm{G}$ systems with randomly deployed users," IEEE Signal Processing Lett., vol. 21, no. 12, pp. 15011505, Sep. 2014.

[3] Z. Ding, F. Adachi, and H. V. Poor, "The application of MIMO to nonorthogonal multiple access," IEEE Trans. Wireless Commun., vol. 15, no. 1, pp. 537-552, Jan. 2016.

[4] Z. Ding, M. Peng, and H. V. Poor, "Cooperative non-orthogonal multiple access in 5G systems," IEEE Commun. Lett., vol. 19, no. 8, pp. 1462 $1465,2015$.

[5] M. Al-Imari, P. Xiao, M. A. Imran, and R. Tafazolli, "Uplink nonorthogonal multiple access for 5G wireless networks," in Proc. 11th International Symposium on Wireless Communications Systems (ISWCS), Barcelona, Spain, Aug 2014, pp. 781785.

[6] B. Kim, W. Chung, S. Lim, S. Suh, and J. Kwun, "Uplink NOMA with Multi-Antenna," in Proc. IEEE Vehicular Technology Conference (VTC Spring), pp. 1-5, May. 2015.

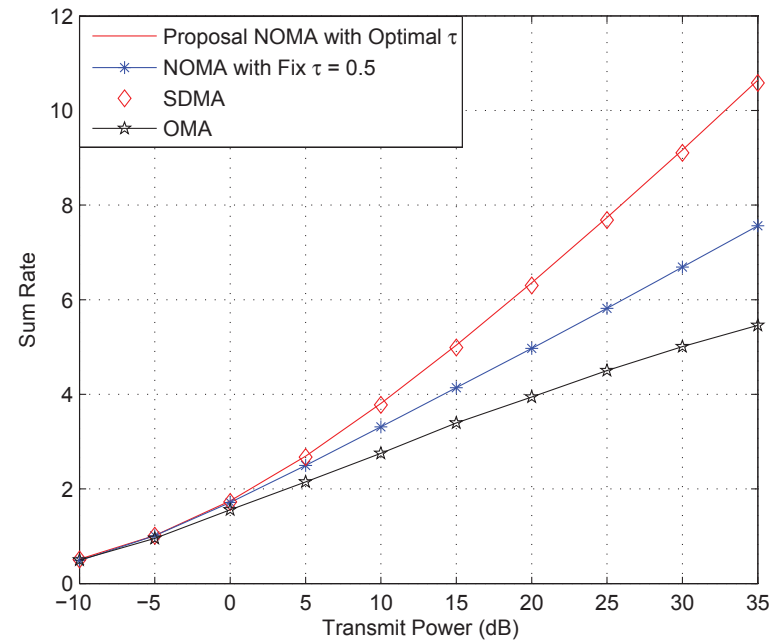

Fig. 3. Sum Throughput variation versus transmission power.

[7] L. R. Varshney, "Transporting Information and energy simultaneously," in Proc. IEEE International Symposium on Information Theory (ISIT), Jul. 2008, pp. 16121616.

[8] R. Zhang and C. K. Ho, "MIMO broadcasting for simultaneous wireless information and power transfer," IEEE Trans. Commun., vol. 12, no. 5, pp. 19892001, 2013.

[9] H. Ju and R.Zhang, "Throughput maximization in wireless powered communication networks," Trans. Wireless Commun.,vol. 13, no. 1, pp. 418-428, Jan. 2014.

[10] L. Liu, R. Zhang, and K. C. Chua, "Multi-antenna wireless powered communication with energy beamforming," IEEE Transactions on Communications, vol. 62, no. 12, pp. 4349-4361, December, 2014.

[11] P. D. Diamantoulakis, K. N. Pappi, Z. Ding, G. K. Karagiannidis, "Optimal Design of Non-Orthogonal Multiple Access with Wireless Power Transfer," Available: http://arxiv.org/abs/1511.01291.

[12] A. Beck, A. Ben-Tal, and L. Tetruashvili, "A sequential parametric convex approximation method with applications to nonconvex truss topology design problems,“ J. Global Optim., vol. 47, no. 1, pp. 29-51, 2010.

[13] Z.-Q. Luo and Z. Shuzhong, "Dynamic spectrum management: Complexity and duality," IEEE J. Sel. Topics Signal Process., vol. 2, no. 1, pp. 5773, 2008.

[14] S. Boyd and L. Vandenberghe, Convex Optimization. Cambridge, U.K.: Cambridge Univ. Press, 2004.

[15] B. R. Marks and G. P. Wright, "A general inner approximation algorithm for nonconvex mathematical programs," Oper. Res., vol. 26, no. 4, pp.681683, Jul. 1978.

[16] M. Grant and S. Boyd, "CVX: Matlab software for disciplined convex programming, version 2.1,“ http://cvxr.com/cvx/, Feb. 2015 